\title{
Cortical and Non-Cortical Myoclonus of Creutzfeldt-Jakob Disease
}

\author{
Jui-Cheng Chen ${ }^{a} \quad Y_{i-T i n g ~ H s u}{ }^{a}$ Thomas Shang-Ming Chiou ${ }^{b} \quad$ Ming-Kuei Lu ${ }^{a} d$ \\ Yu-Chin Lin ${ }^{a}$ Chia-Hung Kao ${ }^{c}$ Wei-Shih Huang ${ }^{a, d}$ Chon-Haw Tsaia, d, e \\ ${ }^{a}$ Neuroscience Laboratory, Department of Neurology, ${ }^{b}$ Department of Neurosurgery, ${ }^{\mathrm{c} D e p a r t m e n t}$ of Nuclear \\ Medicine, China Medical University Hospital, ${ }^{\mathrm{d}}$ School of Medicine, College of Medicine, and ${ }^{\mathrm{e}}$ Graduate Institute of \\ Neural and Cognitive Sciences, China Medical University, Taichung, Taiwan, ROC
}

Dear Sir,

The characteristic symptoms of Creutzfeldt-Jakob disease (CJD) include rapidly progressive cognitive decline and myoclonus [1]. Myoclonus in CJD patients is supposed to be associated with the appearance of periodic synchronous discharges (PSDs) on routine electroencephalograms (EEGs) [2]. However, a casual relationship between myoclonus and PSDs has not been established. Here, we report the case of a CJD patient with positive myoclonus and analyze the cortical potentials locked to the myoclonic jerks.

A 75-year-old man developed difficulty in targeting and catching objects with his left hand 3 weeks before visiting our neurological clinic. One month after the onset of initial symptoms, he started experiencing semi-rhythmic jerks in the left hand that were sensitive to pinprick stimuli. In the following 2 months, the patient showed significant memory and cognitive impairment, whereas muscle tone, muscle strength, and deep-tendon reflexes were intact. On examination, alien hand phenomenon without intermanual conflict was noted. The left hand tended to wander independently and grope nearby objects. Cerebrospinal fluid samples were negative for protein 14-3-3. The possibility of disorders such as thyroid function disorder, vitamin $B_{12}$ and folate deficiencies, hepatitis
$B$, vasculitis, and syphilis was excluded by the results of biochemical screening tests. EEGs showed PSDs with the largest amplitude over the central parietal area of the right hemisphere (i.e. C4 and P4; fig. 1a). Magnetic resonance imaging study showed restricted diffusion over the right frontal, parietal, and occipital lobes in diffusionweighted imaging (DWI) (fig. 1b). ${ }^{99 \mathrm{~m} T \mathrm{Tc}-}$ TRODAT-1 revealed a bilateral decrease in striatal uptake values (fig. 1c). The patient's symptoms continuously deteriorated. The patient became bedridden, and showed akinetic mutism and loss of swallowing capabilities at around 2.5 months after the disease onset. According to WHO criteria (1998), probable CJD was diagnosed. The WHO criteria had a diagnostic accuracy of 96.5\% in a follow-up study performed in 313 patients [1]. Protein 14-3-3 was absent in $9.9 \%$ of the patients diagnosed with probable CJD on the basis of the criteria [1].

\section{Electrophysiological Recordings}

The myoclonic jerks were recorded by multi-channel surface electromyography (EMG). Jerk-locked back-averaged EEG recording was performed with three goldplated electrodes $(\mathrm{C} 3, \mathrm{Cz}$, and $\mathrm{C} 4)$ affixed to the scalp. We adopted EMG onset of spontaneous myoclonic jerks from lefthand triceps as the trigger for the EEG back-averaged sampling (sampling rate $2 \mathrm{kHz}$, filtered band pass $0.05-70 \mathrm{~Hz}$ ). Artifact-free EEG epochs from $200 \mathrm{~ms}$ before to $300 \mathrm{~ms}$ after EMG onset were used for averaging. Somatosensory evoked potential (SEP) was also recorded.

\section{Results}

Surface EMG recording revealed semirhythmic bursts for the recorded muscles at a duration of around $60 \mathrm{~ms}$ (fig. 1d). On the basis of the relationship between the left-hand triceps jerks and the EEG signals, four distinct types (A-D) of conditions were characterized (fig. 1e). Type-A jerks had a mean duration of $60.35 \pm 10.11$ ms and mean amplitude of $179.13 \pm 64.12$ $\mu \mathrm{V}$. The mean latency of the jerk onset to the maximal EEG negativity was $24.22 \pm$ $11.07 \mathrm{~ms}$. In comparison to type-A jerks, type-B jerks had a smaller amplitude $(60.86 \pm 40.43 \mu \mathrm{V})$ and similar duration $(56.85 \pm 9.01 \mathrm{~ms})$. No significant EEG potentials were detected by locking the onset of type-C jerks (mean duration $32.45 \pm$ $3.69 \mathrm{~ms}$, mean amplitude $24.3 \pm 3.40 \mu \mathrm{V}$ ). Some PSD were not jerk-locked (type D). No giant SEP was found.

\section{KARGER}

Fax +4161306 1234 E-Mail karger@karger.ch www.karger.com www.karger.com/ene
Dr. Chon-Haw Tsai

Department of Neurology, China Medical University Hospital

2, Yuh-Der Road, Taichung 404, Taiwan (ROC)

Tel. +886 42206 2121, ext. 5035, Fax +886 422344055

E-Mail windymovement@yahoo.com.tw 

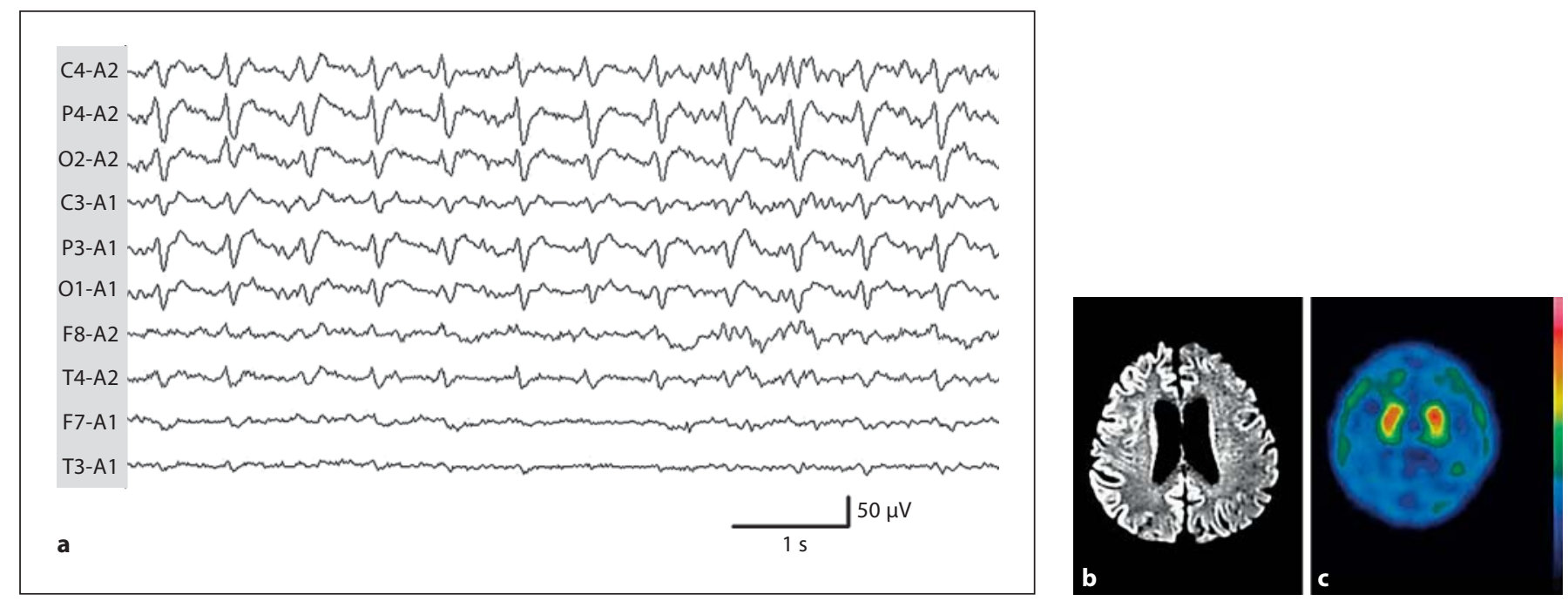

Fig. 1. a Routine EEG study shows PSDs with predominance over the C4 and P4 electrodes. b Increased signal intensity in DWI at right frontal, parietal, and occipital cortical areas. c 99mTc-TRODAT-1 SPECT analysis of the brain shows decreased uptake in right striatum (1.12) and left striatum (1.07). The specific uptake is obtained by subtracting the mean counts per pixel in the occipital cortex (OC) and the mean counts per pixel in the whole striatum (ST) and dividing the result by the mean counts per pixel in the background (equations: (ST - OC)/OC; referential normal values in striatum: 1.692.15). d Surface EMG recorded from the left-hand triceps muscle of the patient. The signals show a semi-rhythmic pattern of the EMG bursts. e Four types of the averaged EMG and EEG recorded from the patient. Type A and B myoclonic jerks are locked to the cortical potentials, most obviously at $\mathrm{C} 4$, and are shown in the first and second rows, respectively. The third row demonstrates the absence of timelocked cortical potentials for some of the myoclonic jerks. The fourth row shows the cortical potentials without time-locked myoclonic jerks. The numbers of the averaged EEG epochs are 193, 43, 129 and 114 for types A, B, C, and D, respectively.
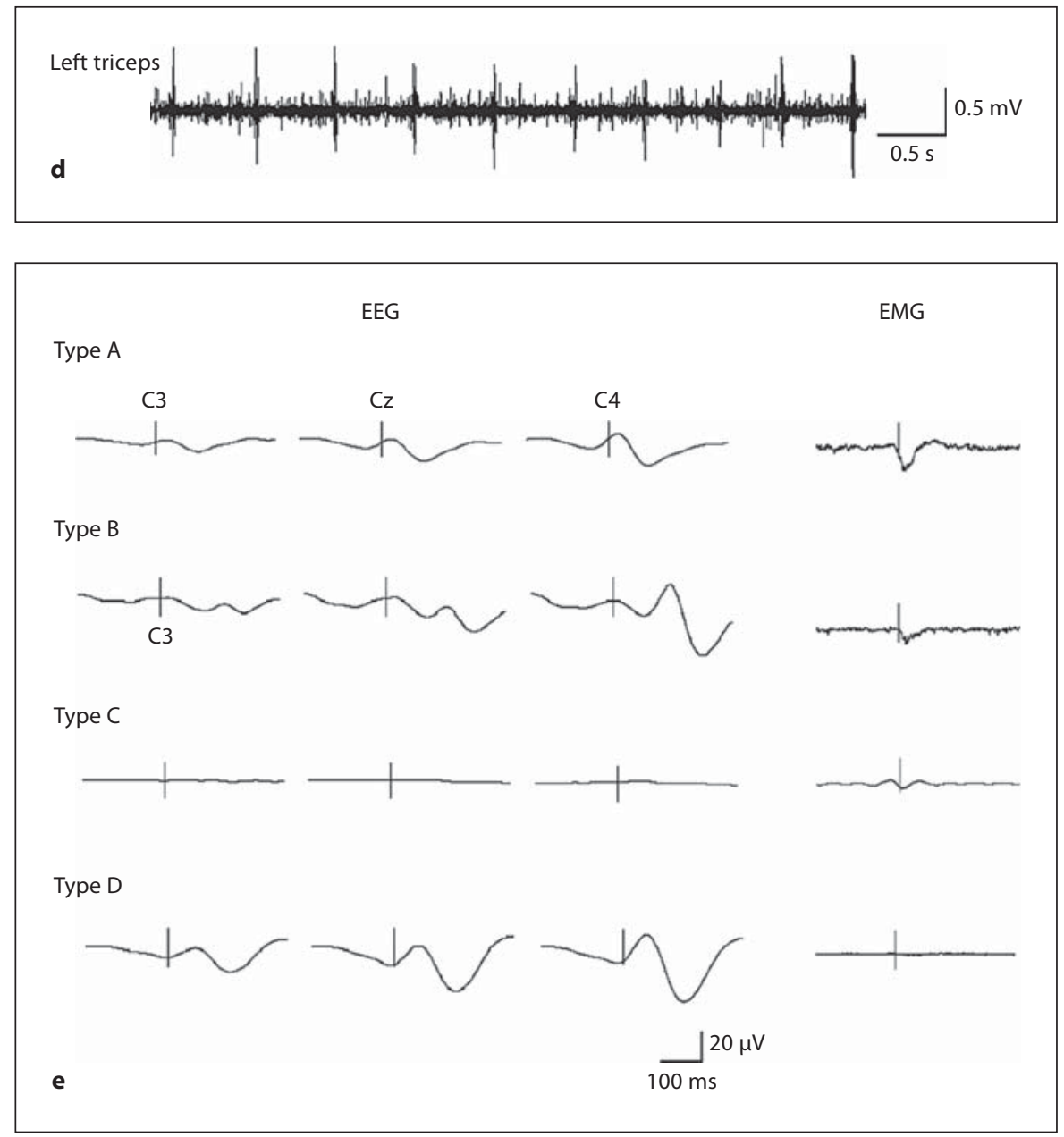
PSD is a common and characteristic EEG finding in CJD patients [3]. An abnormal subcortical pacemaker, which is probably synchronized with the cortical potentials, was supposed to play a pivotal role in the PSD generation [4]. Impairment of normal inhibitory function at the cortical level may further amplify the influence of this pathological pacemaker [4]. Myoclonus is another important symptom for the diagnosis of CJD and often diffuse, generalized and relatively rhythmic in CJD patients. In asymmetric cases, the jerks are usually accompanied by dystonia or alien hand syndrome [5]. Both positive and negative myoclonus have been reported in CJD. Some cases did not show a giant SEP or enhanced $C$ reflex [6]. To which extent the observed PSD in CJD patients is relevant to the myoclonus remains unclear. Shibasaki et al. [7] employed a jerk-locked SEP technique and reported that cortical excitability was suppressed between periodic myoclonic jerks, and this suppression was associated with PSD in a CJD patient. Our findings for the current CJD patient provide further insight into this phenomenon and illustrate that the cortical potentials may or may not lock to the myoclonic jerks and vice versa. One of the possible explanations for this finding is that the generators of the myoclonic jerks are located at different cortical and subcortical levels. The fact that some jerks were not associated with any detectable cortical potentials may indicate that their generator was located very far away from the cortex, i.e. subcortically located, and such jerks were unable to be recorded during the current scalp EEG recording; alternatively, these jerks were randomly evoked and not locked to any specific cortical potentials in a fixed time period. In a typical subcortical myoclonus, i.e. reticular reflex or hyperekplexia, the jerks may involve all four limbs and the muscles innervated by the cranial nerve. The jerk duration may range from 50 to $100 \mathrm{~ms}$ [8], which is longer than the duration of the type- $C$ jerks observed in the present study. In contrast, the appearance of robust cortical potentials or PSDs was not always restricted to any myoclonic jerks. These findings support the notion that the generalized PSD may have different influences on the motor neuron excitabilities at cortical and subcortical levels. The subcortical involvement in the current patient was also evidenced by the bilaterally decreased striatal uptake (fig. 1c), although this phenomenon may be irrelevant to the generation of jerks or PSDs.

The direct evidence that confirmed the cortical origin of type-A and -B jerks was the presence of a jerk-locked EEG event.
The latency between the onset of jerks and the peak of the cortical potentials was $\sim 24$ $\mathrm{ms}$, which is close to the physiological range of the SEP. In the cases of jerk-locked EEG events, we did not find giant SEPs in the scalp recordings. Giant SEPs were classically found in patients with cortical myoclonus [9-11]. However, giant SEP was not seen in all patients with cortical myoclonus. This finding implied that either these patients have no abnormality in the cortical processing of sensory inputs, or the dysfunction is not sufficient to be detected by classical SEP studies [12]. For example, there were usually no giant SEPs in corticobasal degeneration patients with cortical myoclonus. This may result from cortical lesions with functional loss in the corresponding area $[13,14]$ and this could also be the reason in the current CJD patient who showed a lateralizing cortical involvement in DWI (fig. 1b).

\section{Acknowledgements}

The authors are thankful to the Taiwan Creutzfeldt-Jakob Disease Committee and Research Group for their help in the disease diagnosis. The present report is supported by grants from DMR-99-175 and NSC99-2314-B-039-016-MY2.

\section{References}

1 Zerr I, Pocchiari M, Collins S, Brandel JP, de Pedro Cuesta J, Knight RS, Bernheimer H, Cardone F, Delasnerie-Laupretre N, Cuadrado Corrales N, Ladogana A, Bodemer M, Fletcher A, Awan T, Ruiz Bremon A, Budka H, Laplanche JL, Will RG, Poser S: Analysis of EEG and CSF 14-3-3 proteins as aids to the diagnosis of Creutzfeldt-Jakob disease. Neurology 2000;55:811-815.

-2 Shibasaki H, Motomura S, Yamashita Y, Shii H, Kuroiwa Y: Periodic synchronous discharge and myoclonus in Creutzfeldt-Jakob disease: diagnostic application of jerk-locked averaging method. Ann Neurol 1981;9:150156.

3 Chiofalo N, Fuentes A, Galvez S: Serial EEG findings in 27 cases of Creutzfeldt-Jakob disease. Arch Neurol 1980;37:143-145.
-4 Traub RD, Pedley TA: Virus-induced electronic coupling: hypothesis on the mechanism of periodic EEG discharges in Creutzfeldt-Jakob disease. Ann Neurol 1981; 10:405-410.

$\checkmark 5$ Maltete D, Guyant-Marechal L, Mihout B, Hannequin D: Movement disorders and Creutzfeldt-Jakob disease: a review. Parkinsonism Relat Disord 2006;12:65-71.

6 Ohnari K, Matsunaga K, Uozumi T, Tamagawa A, Hashimoto T, Tsuji S: Unilateral positive-negative myoclonus in Creutzfeldt-Jakob disease. Mov Disord 2006;21:1963-1966.

-7 Shibasaki H, Neshige R, Hashiba Y: Cortical excitability after myoclonus: jerk-locked somatosensory evoked potentials. Neurology 1985;35:36-41.

$\checkmark 8$ Tsai CH, Chang FC, Su YC, Tsai FJ, Lu MK, Lee CC, Kuo CC, Yang YW, Lu CS: Two novel mutations of the glycine receptor gene in a Taiwanese hyperekplexia family. Neurology 2004;63:893-896. $\checkmark 9$ Obeso JA, Rothwell JC, Marsden CD: Somatosensory evoked potentials in myoclonus. Adv Neurol 1986;43:373-384.

$>10$ Kakigi R, Shibasaki H: Generator mechanisms of giant somatosensory evoked potentials in cortical reflex myoclonus. Brain 1987;110:1359-1373

11 Mima T, Nagamine T, Nishitani N, Mikuni $\mathrm{N}$, Ikeda A, Fukuyama $\mathrm{H}$, Takigawa $\mathrm{T}$, Kimura J, Shibasaki H: Cortical myoclonus: sensorimotor hyperexcitability. Neurology 1998;50:933-942

12 Cassim F, Houdayer E: Neurophysiology of myoclonus. Neurophysiol Clin 2006;36:281291.

13 Thompson PD: Myoclonus in corticobasal degeneration. Clin Neurosci 1995;3:203208.

14 Carella F, Ciano C, Panzica F, Scaioli V: Myoclonus in corticobasal degeneration. Mov Disord 1997;12:598-603. 